\title{
Nghiên cứu thiết kế và chế tạo máy in 3D bê tông
}

\author{
Lê Cao Chiến ${ }^{1 *}$, Nguyễn Văn Tuấn ${ }^{2}$, Lê Trung Thành ${ }^{1}$, Phùng Thị Mai Phương ${ }^{1}$ \\ ${ }^{1}$ Viện Vật liệu Xây dựng, Số 235 Nguyễn Trãi, P. Thanh Xuân Trung, Q. Thanh Xuân, TP. Hà Nội \\ ${ }^{2}$ Khoa Cơ khí - Cơ điện tử, Trường Đại học Phenikaa
}

\begin{tabular}{l} 
TỪ KHOÁ \\
\hline Bê tông \\
Đầu in \\
Điều khiển \\
In 3D
\end{tabular}

KEYWORDS

Concrete

Nozzle

Control

3D printing

\begin{abstract}
TÓM TẮT
Công nghệ in 3D bê tông hiện nay là đại diện điển hình của ngành xây dựng về việc áp dụng công nghệ của Cách mạng công nghiệp lần thứ tư vào kỹ thuật xây dựng hiện đại. Để có thể hiện thực hoá kỹ thuật in 3D bê tông trong chế tạo các cấu kiện vừa có khả năng chịu lực tốt vừa có hình dáng kiến trúc, thẩm mỹ đẹp thì máy in 3D bê tông chính là công cụ quan trọng nhất. Bài báo này trình bày kết quả nghiên cứu thiết kế và chế tạo một máy in 3D bê tông cỡ lớn điển hình có kích thước tổng thể 4,92 x 2,53 x 2,53 m và kích thước cấu kiện lớn nhất có thể in được lên đến 1,8 x 1,8 x 2,5 m. Đây là sản phẩm máy in 3D bê tông đầu tiên của Việt Nam.
\end{abstract}

\section{Giới thiệu}

Lịch sử phát triển xây dựng kết cấu công trình sử dụng vật liệu bê tông cho thấy có một hạn chế kỹ thuật cố hữu đó là phải sử dụng hệ thống ván khuôn để đổ hỗn hợp bê tông vào và phải chờ cho bê tông rắn chắc, có khả năng chịu lực, sau đó mới được dỡ ván khuôn ra. Nhược điểm này ngày càng bộc lộ khi mà các kiến trúc sư thiết kế kết cấu công trình với nhiều ý tưởng độc đáo và có tính thẩm mỹ cao làm cho công tác chuẩn bị ván khuôn đổ bê tông khó có thể thực hiện được và rất tốn thời gian cũng như chi phí khi sử dụng máy móc thiết bị và tay nghề công nhân hiện nay. Các hạn chế cố hữu của kỹ thuật xây dựng với vật liệu bê tông hoàn toàn có thể được giải quyết bằng công nghệ in $3 \mathrm{D}$ bê tông. In $3 \mathrm{D}$ là một kỹ thuật chế tạo đắp lớp (Additive manufacturing technique), trong đó vật liệu được kết nối với nhau theo từng lớp để hình thành nên vật thể cần gia công từ dữ liệu mô hình thiết kế 3D [1-7]. Quá trình bắt đầu với mô hình thiết kế 3D bằng phần mềm máy tính. Sau đó mô hình này được chuyển sang định dạng ngôn ngữ phân vùng không gian tiêu chuẩn (STL - Standard Tessellation Language) đưa ra các thông tin về tọa độ và ranh giới của các đối tượng cần chế tạo. Tiếp đến các đối tượng in đã được phân vùng không gian này được cắt thành các lớp với chiều dầy các lớp bằng nhau. Sau đó, các lớp được chuyển đổi thành mã G-code là các tập tin chứa thông tin đường in để điều khiển máy in chế tạo vật thể. Lưu đồ quy trình để tiến hành in 3D được thể hiện như trong Hình 1.
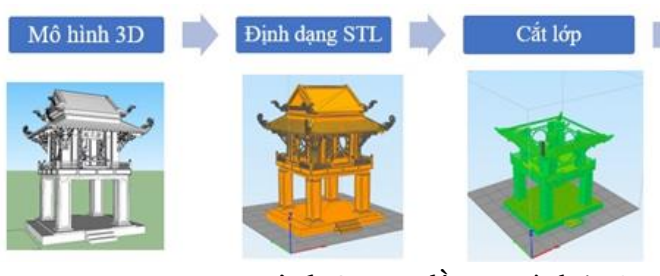

Hình 1. Lưu đồ quy trình in 3D.

Công nghệ in 3D bê tông đã được nghiên cứu phát triển tại Mỹ từ năm 1998 theo kiểu kỹ thuật in theo đường viền (Contour Crafting) [6] bằng kỹ thuật ép đùn hỗn hợp bê tông. Sử dụng phương pháp này có thể tạo ra các bức tường uốn cong hay các vật thể họa tiết cầu kỳ có kết cấu vững chắc hơn so với phương pháp xây dựng truyền thống. Độ mịn của quá trình ép đùn vật liệu đạt được bằng cách kiểm soát độ chảy của đầu đùn theo hướng dọc và ngang. Tuy nhiên, công nghệ này có những hạn chế là: bị giới hạn trong ép đùn dọc, do đó mang lại các cấu trúc liên kết 2.5D (mở rộng theo chiều dọc của hình phẳng); Cấu trúc 3D tạo viền có vai trò như "ván khuôn" ban đầu có thể khá phức tạp để thực hiện khi sản xuất, tùy thuộc vào kích thước và hình dạng của vật thể được in; Việc đúc bê tông tuần tự bị gián đoạn trong "ván khuôn" do áp suất thủy tĩnh và tính chất cơ học yếu của bê tông đùn có thể dẫn đến các vùng liên kết yếu giữa các lớp. 


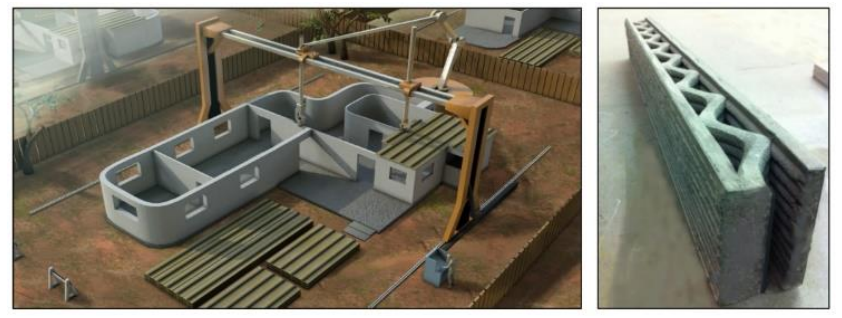

Hình 2. Mô hình kỹ thuật Contour Crafting in đường viền kết cấu và sản phẩm điển hình.

Từ những năm 2000, Đại học Loughborough, Vương Quốc Anh đã nghiên cứu phát triển kỹ thuật in 3D bê tông (3D Concrete Printing) dựa trên nguyên tắc ép đùn, tương tự như chế tạo đường viền của công nghệ Contour Crafting, đồng thời có thể thực hiện in các đường viền phức tạp và liên kết chặt chẽ với nhau. Chính nhờ đặc điểm này, $3 \mathrm{D}$ Concrete Printing được xem là giải pháp tiềm năng nhất trong xây dựng hiện nay bởi vì công nghệ này có thể chế tạo nhanh ra các tòa nhà/công trình đồng dạng, hàng loạt cũng như in ra các cấu kiện, ngôi nhà có kiến trúc khác nhau. Trong công nghệ này, hỗn hợp bê tông được vận chuyển qua một hệ thống ống và bơm được điều khiển tự động, cuối cùng được đùn qua một vòi phun để in các lớp bê tông. Đây là một quy trình sản xuất liên tục, trong đó, các lớp vật liệu đùn ướt liên tiếp được liên kết với nhau theo tầng $[5,8]$. Phương pháp này có thể tạo ra các bề mặt tiếp xúc liên tục giữa hai lớp bê tông in. Do đó, sẽ tránh được các khoảng trống hình học giữa hai lớp trong kỹ thuật in 3D [9, 10].
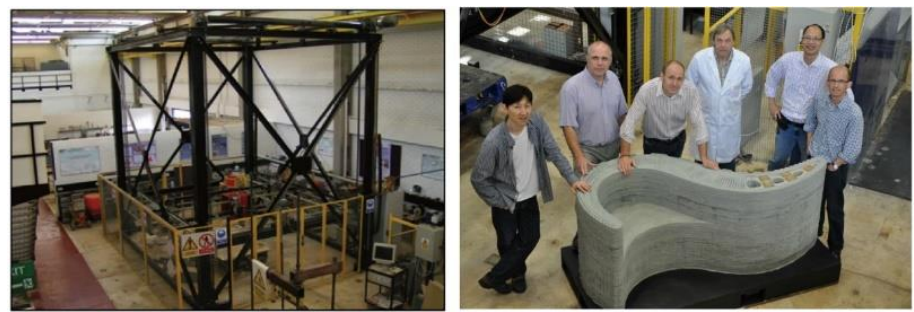

Hình 3. Kỹ thuật in 3D bê tông (3D Concrete Printing) và sản phẩm có đường cong [5].

Trong các quy trình in đắp lớp, khả năng bơm và tính ổn định của quá trình đùn là rất quan trọng. Đặc tính này sẽ khác nhau tùy thuộc vào thành phần hỗn hợp bê tông, hệ thống phân phối và đầu in. Thông số kỹ thuật chung trong quá trình in 3D bê tông được phân loại thành bốn đặc tính chính bao gồm [11]:

- Khả năng bơm: Hỗn hợp bê tông di chuyển qua hệ thống phân phối dễ dàng và tin cậy.

- Khả năng in: Sợi bê tông in ra giữ được hình dáng ổn định và liên tục.

- Khả năng xây: Khả năng chống biến dạng của các lớp bê tông, được tạo ra bởi các sợi bê tông in liên kết với nhau, dưới tác dụng của tải trọng bản thân và tải trọng của các lớp bê tông phía trên in ra sau.

- Thời gian thi công: Khoảng thời gian mà hỗn hợp bê tông giữ được khả năng bơm, khả năng in và khả năng xây trong dung sai có thể chấp nhận được.
Giai đoạn in thực hiện bởi máy in 3D là một hệ thống giàn dẫn động một bộ đầu in được điều khiển để định vị đầu in theo tọa độ Descartes XYZ (Hình 4). Sự phối hợp giữa tốc độ di chuyển của đầu in và tốc độ dòng nguyên liệu được đùn ra là rất quan trọng để tạo ra đường in mong muốn.

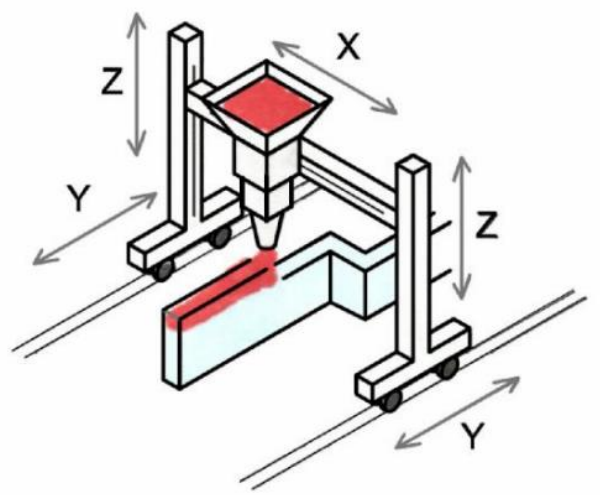

Hình 4. Mô tả cấu trúc máy in 3D.

Áp dụng công nghệ in 3D bê tông trong xây dựng tại Việt Nam nhìn chung vẫn còn chậm do thiếu các loại máy in 3D thương mại với giá cả phù hợp. Vì vậy, việc nghiên cứu thiết kế và chế tạo máy in 3D bê tông khổ lớn là yêu cầu cấp thiết, nhằm phát triển và làm chủ công nghệ in 3D bê tông phù hợp với điều kiện kinh tế, kỹ thuật tại Việt Nam. Bài báo này trình bày kết quả nghiên cứu thiết kế và chế tạo máy in 3D bê tông có mô hình như trong Hình 5 để in kết cấu có kích thước tối đa $1,8 \mathrm{~m} \mathrm{x} \mathrm{1,8} \mathrm{m} \mathrm{x} \mathrm{2,5} \mathrm{m.} \mathrm{Động} \mathrm{cơ} \mathrm{bước} \mathrm{có} \mathrm{tốc} \mathrm{độ} 3000$ vòng/phút được lựa chọn là cơ cấu chấp hành trên tất cả các trục để tối ưu về chi phí và cung cấp chức năng mong muốn. Động cơ bước có mô-men xoắn cao cho phép máy in thực hiện các chuyển động tốc độ cao cần thiết khi in phun. Mỗi động cơ được ghép nối với trình điều khiển bước hoạt động ở chế độ vi bước (microstepping) cho phép động cơ bước dừng và định vị tại vị trí nửa bước giữa 2 bước đủ. Ưu điểm của phương pháp này là động cơ có thể hoạt động với góc bước nhỏ, độ chính xác cao. Do xung cấp có dạng sóng nên động cơ hoạt động êm hơn, hạn chế được vấn đề cộng hưởng khi động cơ hoạt động.

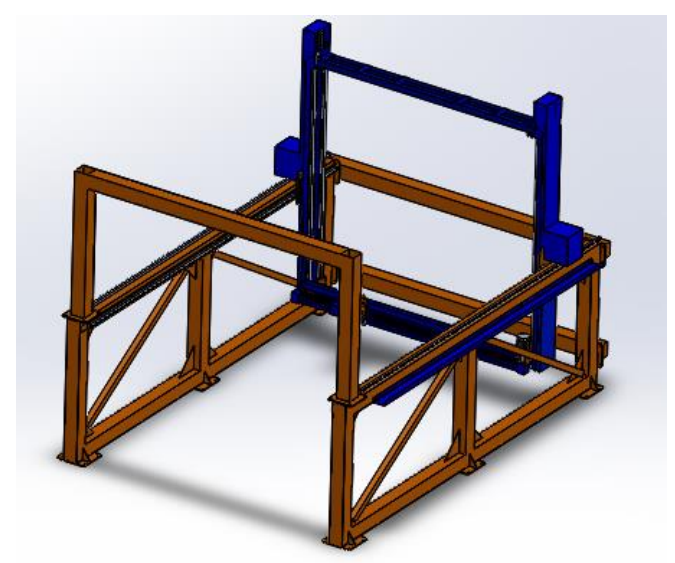

Hình 5. Mô hình máy in 3D bê tông được nghiên cứu thiết kế và chế tạo. 


\section{Thiết kế và chế tạo khung cho máy in 3D}

Khung cơ khí được thiết kế đảm bảo yêu cầu kỹ thuật như sau: Dung sai độ phẳng: $0,03 \mathrm{~mm} / \mathrm{chiều} \mathrm{dài,} \mathrm{Dung} \mathrm{sai} \mathrm{độ} \mathrm{phẳng} \mathrm{bề} \mathrm{mặt:} 0,01$ $\mathrm{mm}$, Dung sai giữa các bề mặt song song: $0,05 \mathrm{~mm} /$ chiều dài, Dung sai độ vuông góc: $0,05 \mathrm{~mm}$; Sai số ví trị tương quan khi lắp ráp theo chiều dài: $0,1 \mathrm{~mm}$.

Các cụm trục $\mathrm{X}, \mathrm{Y}, \mathrm{Z}$ được truyền động độc lập có thể tháo rời để linh hoạt trong việc lắp đặt và vận chuyển máy in. Cụm trục $\mathrm{X}$ có chiều dài hành trình là $1.800 \mathrm{~mm}$. Trục này là trục mang đầu in chứa vật liệu nên trục này sẽ chịu tải trọng võng và được xem là trục yếu nhất trong ba trục của máy in. Để hạn chế vấn đề cong võng, không gây ảnh hưởng đến độ chính xác khi in, trục $\mathrm{X}$ được thiết kế sử dụng bộ truyền động thanh răng - bánh răng nhằm không gây ra độ trượt, độ rung cho phép truyền động một lực vừa đủ. Đầu đùn được gắn và di chuyển dọc theo trục $\mathrm{X}$ bởi hai thanh ray định hướng để hạn chế chuyển động qua lại, tránh các rung động và biến dạng có thể xảy ra trong quá trình chuyển động của máy in như trong Hình 6.

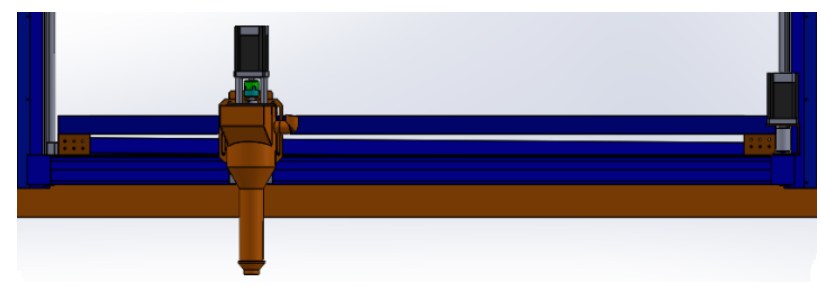

Hình 6. Bản vẽ thiết kế trục $X$.

Trục $\mathrm{X}$ được gắn với trục $\mathrm{Z}$ để di chuyển lên xuống theo phương $\mathrm{Z}$. Trục này chịu toàn bộ tải trọng của trục $\mathrm{X}$ và đầu đùn để tăng độ ổn định, chuyển động êm với độ chính xác cao. Trục $\mathrm{Z}$ được thiết kế sử dụng cơ cấu truyền động vitme bi với hành trình $1.800 \mathrm{~mm}$. Gắn với trục $X$ thông qua 4 trục định hướng đặt hai bên và cố định vào khung. Hình 7 thể hiện bản vẽ thiết kế trục $Z$ (gồm có 2 trục $Z 1$ và $Z 2$ thiết kế như nhau đặt mỗi bên).

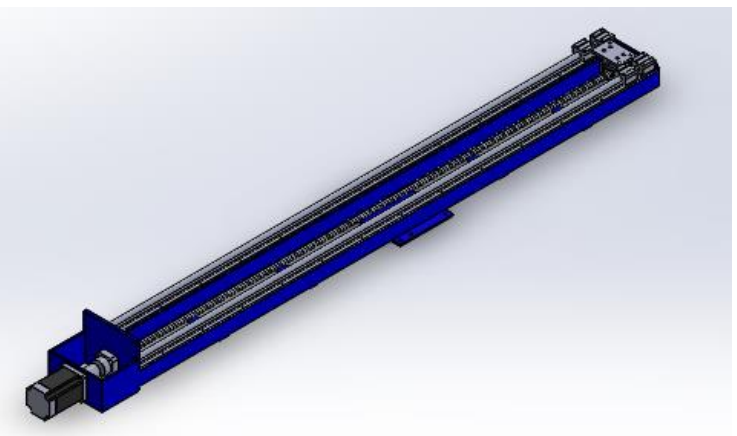

Hình 7. Bản vẽ thiết kế trục Z.

Trục Y là trục chuyển động được thiết kế có hành trình dài nhất $3.000 \mathrm{~mm}$. Đây là trục chịu lực chính cho toàn bộ hệ thống máy in, chịu tải trọng của toàn bộ trục $\mathrm{X}$ và $\mathrm{Z}$ cũng như đầu in. Vì vậy để hạn chế vấn đề cong võng, không gây ảnh hưởng đến độ chính xác khi in, trục $\mathrm{Y}$ được thiết kế sử dụng bộ truyền động thanh răng - bánh răng nhằm không gây ra độ trượt, độ rung. Trục $\mathrm{Y}$ được thiết kế lớn nhất và chắc chắn nhất trong hệ thống máy in, được gia cố bằng hệ khung chịu lực để tăng độ vững chắc cho máy in. Hình 8 thể hiện bản vẽ thiết kế trục Y.

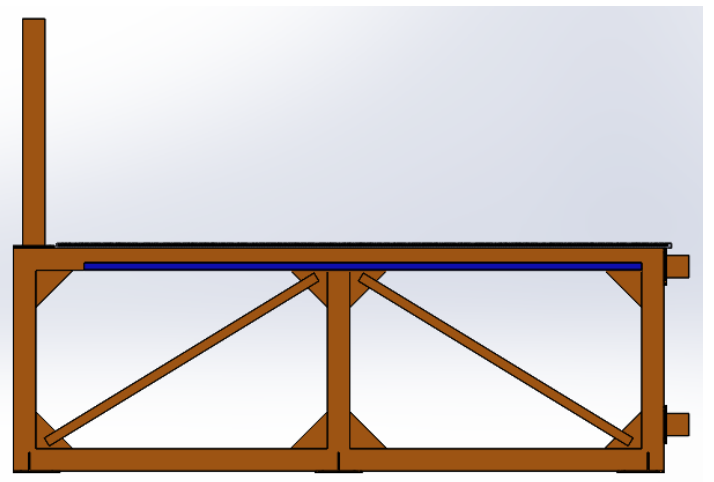

Hình 8. Bản vẽ thiết kế trục Y.

\section{Thiết kế và chế tạo đầu in bê tông}

In 3D bê tông là một quá trình dựa trên nguyên tắc đùn hỗn hợp vật liệu bê tông tươi. Hỗn hợp được lưu trữ trong bồn chứa và vận chuyển qua đầu cấp liệu, cuối cùng được đùn qua đầu in để tạo thành các lớp bê tông in được thể hiện trên Hình 9. Có ba cơ cấu chính có thể được sử dụng để dẫn dòng hỗn hợp bê tông qua đầu in: Cơ cấu trục vít, cơ cấu pittong và cơ cấu khí nén như minh họa trong Hình 10. Cơ cấu trục vít có ưu điểm là hỗn hợp bê tông được giữ chuyển động liên tục và được sử dụng phổ biến trong các đầu in $3 \mathrm{D}$ bê tông. Vấn đề chính của cơ cấu này là phải thiết lập mối quan hệ trực tiếp giữa chuyển động quay của trục vít và chuyển động của đầu in theo các trục $\mathrm{X}, \mathrm{Y}, \mathrm{Z}$. Cơ cấu đùn sử dụng áp suất không khí hoặc sử dụng pittong để ép hỗn hợp bê tông tươi qua đầu đùn cũng đã được chứng minh là những phương pháp hiệu quả, áp suất đùn phải được hiệu chỉnh theo chuyển động của đầu in. Tuy nhiên khi xem xét đồng thời các yếu tố như độ phức tạp, thuận tiện trong sản xuất, khả năng tạo áp lực thì cơ cấu trục vít được đánh giá cao hơn [13].

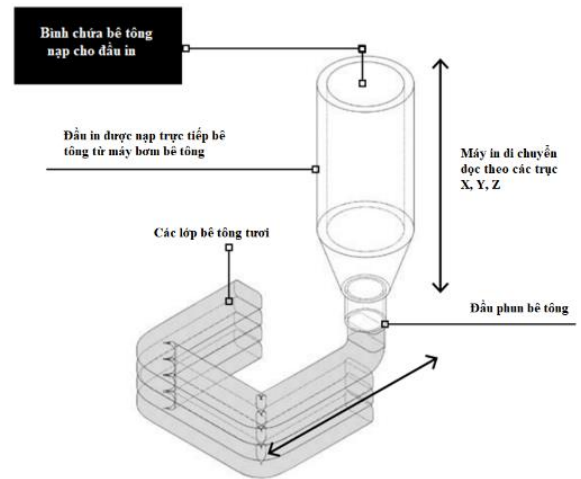

Hình 9. Nguyên tắc in 3D bê tông. 


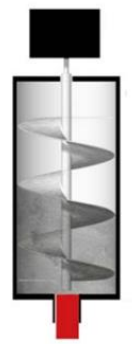

a) Cơ cấu trục vít

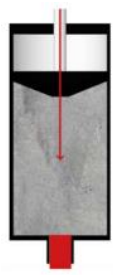

b) Cơ cấu pittong

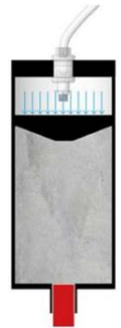

c) Cơ cấu khí nén

Hình 10. Các loại cơ cấu được sử dụng trong ép đùn trong in 3D bê tông.

Đầu in bê tông được thiết kế dạng trụ, đường kính 2 cấp để chứa vật liệu trước khi vít tải chuyển hỗn hợp bê tông đến đầu in. Bộ phận này được điều khiển bằng 1 động cơ servo có điều khiển tốc độ quay. Có thể tiếp liệu bằng tay hoặc tự động thông qua một máy bơm bê tông. Đầu in được thiết kế tháo lắp dễ dàng để có thể thay đổi biên dạng đầu in khi cần thiết, xem Hình 11. Cụm đầu in được lắp với trục X bằng các bu lông.
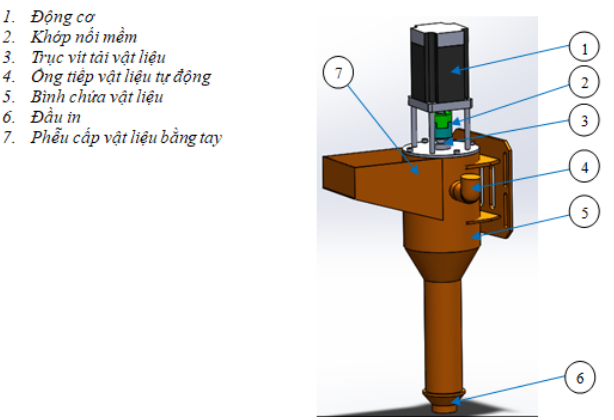

Hình 11. Thiết kế cụm đầu in.

\section{Thiết kế và chế tạo hệ thống điện và điều khiển \\ 4.1. Mạch điều khiển}

Bo mạch điều khiển được tạo thành từ hai thành phần: Bộ vi điều khiển và bảng mạch. Các thành phần này có thể được kết hợp thành một bảng mạch hoặc thành dạng mô đun riêng biệt được gắn vào nhau. Cả hai đều hoạt động đồng thời để kiểm soát và phân phối điện năng cho tất cả các thành phần hệ thống điều khiển. Đây được coi là bộ não và hệ thống thần kinh trung ương của máy in.

Các động cơ bước của máy in 3D cần được điều khiển chính xác để tạo ra vật thể kết cấu bê tông in chất lượng. Một trong những cấu phần chịu trách nhiệm chính là trình điều khiển động cơ bước. Trình điều khiển động cơ bước là một chip điều khiển dòng điện đến động cơ bước để động cơ có thể được định vị chính xác. Các trình điều khiển nhận được tín hiệu từ bộ vi điều khiển chỉ định động cơ bước cần chuyển động bao nhiêu vòng. Thông thường, bộ vi điều khiển không cung cấp nguồn trực tiếp cho động cơ, vì bộ vi điều khiển không thể cung cấp đủ năng lượng để điều khiển động cơ bước. Sử dụng trình điều khiển, bộ vi điều khiển có thể kiểm soát tốc độ và vị trí của động

cơ bước trong khi nguồn cấp cho động cơ trực tiếp từ nguồn điện. Trình điều khiển động cơ thường có sẵn ở dạng mạch tích hợp (IC). Mạch điện tử, trình điều khiển động cơ hoạt động như bộ khuếch đại dòng vì chúng lấy một tín hiệu nhỏ từ bộ điều khiển và tạo ra đầu ra giống tín hiệu đầu vào nhưng dòng cao hơn. Hệ thống này cho phép động cơ hoạt động ở dòng điện cao sẽ không làm hỏng bo mạch điều khiển.

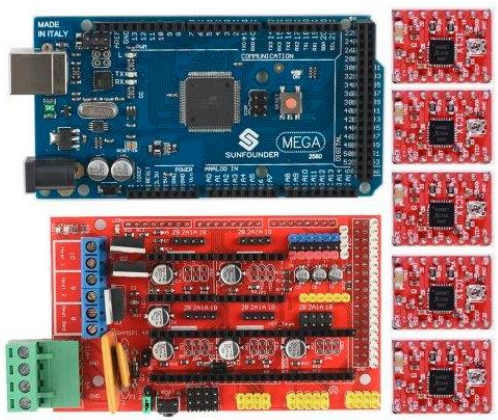

Hình 12. Bo mạch điều khiển.

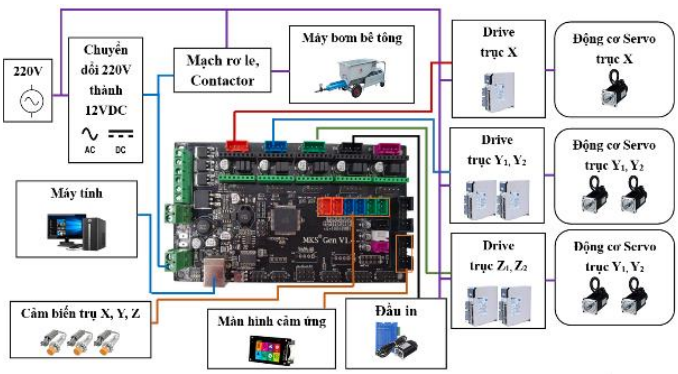

Hình 13. Sơ đồ mạch điều khiển máy in $3 \mathrm{D}$ bê tông.

\subsection{Phần mềm điều khiển}

Hệ thống điều khiển cho máy in 3D bao gồm hai thành phần chính: phần mềm điều khiển (firmware) và phần mềm cắt lớp. Phần mềm điều khiển được tích hợp vào bo mạch chủ của máy in, có chức năng dịch hiểu mã G-code và gửi các lệnh tương ứng đến các thành phần của máy in để điều khiển các trục $X, Y, Z$, biên dịch mã G-code thành xung điện để điều khiển các động cơ hoạt động. Các chương trình firmware cho máy in 3D mã nguồn mở thông dụng như: Marlin, Sprinter, Repetier...

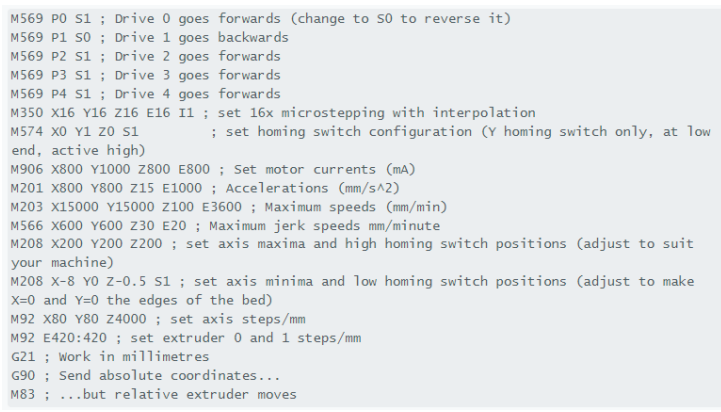

Hình 14. Khai báo cấu hình RepRapFirmware cho máy in tọa độ Descartes. 
Phần mềm cắt, được cài đặt trên máy tính cá nhân, tạo G-code cho chương trình điều khiển máy in [14]. Qui trình chế tạo một thành phần sử dụng kỹ thuật in $3 \mathrm{D}$ nói chung bắt đầu từ file mô hình $3 \mathrm{D}$ có thể được vẽ trên các phần mềm vẽ $3 \mathrm{D}$ chuyên dụng như AUTOCAD, SOLIDWORD, SKETCHUP,... Tập tin 3D sẽ được chuyển đổi sang định dạng mà máy in 3D có thể hiểu và nhận dạng thường là định sạng STL. Các file định dạng STL chứa thông tin về mỗi lớp in được gửi đến phần mềm cắt lớp. Phần mềm cắt lớp cho phép quản lý mô hình STL, thực hiện các điều chỉnh nhỏ đối với hình dạng của một phần, sửa lỗi, thay đổi hướng các bộ phận, chỉ định độ dày lớp và lượng vật liệu đùn, chất lượng in, thay đổi các thông số gia công, mô phỏng quá trình và tạo đường in. Tất cả các lỗi có thể xảy ra trên mô hình in 3D đều có thể được sửa và điều chỉnh trong bước này. Khi tất cả các thao tác với mô hình STL được thực hiện, chương trình cắt lớp sẽ tạo ra mã G-code. Gcode được tải lên trực tiếp từ PC bằng giao diện Web, cáp hoặc thẻ nhớ.

\section{Chế tạo và lắp đặt máy in 3D bê tông}

Trên cơ sở nghiên cứu thiết kế, máy in 3D bê tông đầu tiên của Việt Nam đã được chế tạo thành công (Hình 15) với kích thước tổng thể 4,92 x 2,53 x 2,53 m, tốc độ in tối đa $250 \mathrm{~mm} / \mathrm{s}$, tốc độ phun hỗn hợp bê tông đạt tối đa 50 lít/phút để có thể in được kết cấu có kích thước tối đa 1,8 m x 1,8 m x 2,5 m. Máy in 3D bê tông có hệ kết cấu rất chắc chắn và cân đối, không có bộ phận nào của máy in bị rung và biến dạng. Các bài kiểm tra chuyển động cho thấy tính toàn vẹn của việc lắp đặt máy in và thành công của thiết kế được phát triển. Phần cơ khí, hệ thống điện và phần mềm điều khiển máy cũng đã được triển khai lắp đặt và phát triển đồng bộ để đảm bảo máy in 3D bê tông hoạt động hoàn toàn ổn định.

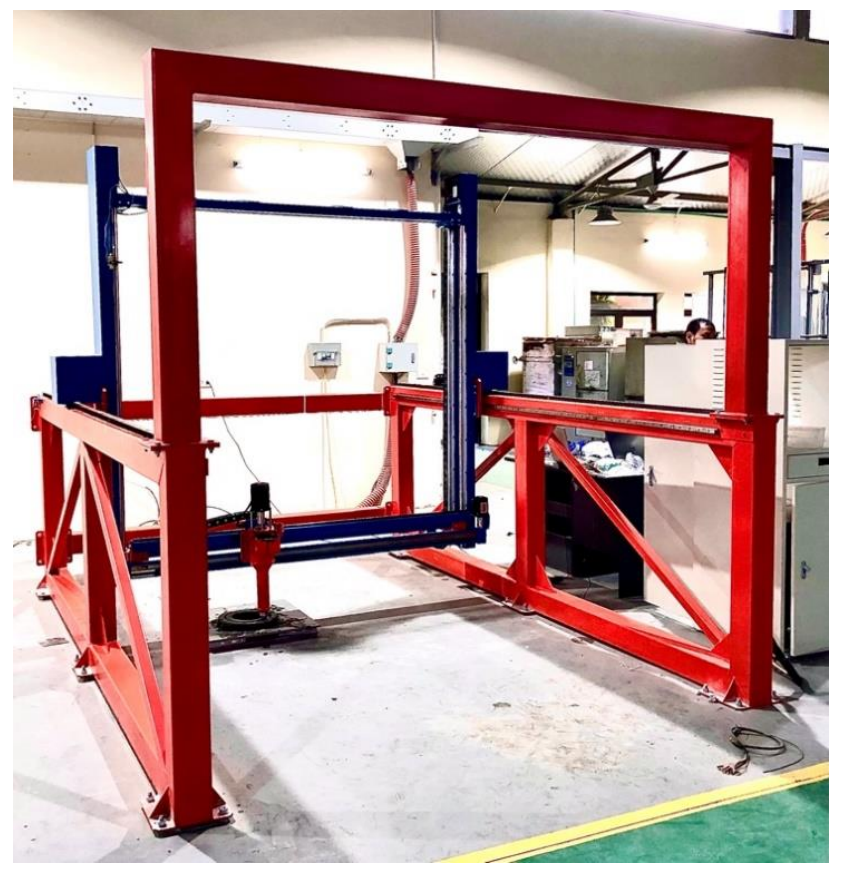

a) Máy in $3 D$ bê tông

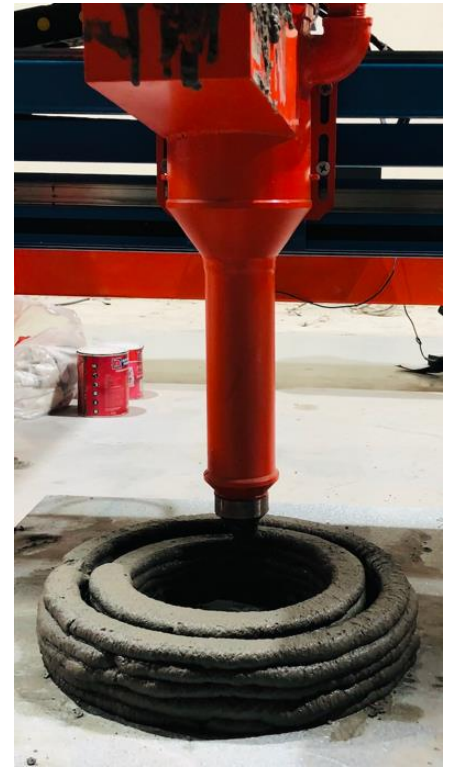

b) Mẫu in thử

Hình 15. Hệ thống máy in 3D bê tông đầu tiên của Việt Nam được thiết kế và chế tạo tại Viện Vật liệu xây dựng, Bộ Xây dựng.

\section{Kết luận}

Công nghệ in 3D bê tông đang trở thành công nghệ hiện đại có khả năng ứng dụng rộng rãi trong ngành xây dựng. Trong vài thập kỷ vừa qua, công nghệ in 3D bê tông đã có bước tiến vượt bậc và đã chứng minh là công nghệ đáng tin cậy để chế tạo được các sản phẩm kết cấu công trình với tính thẩm mỹ, kiến trúc cao, thân thiện với môi trường. Công nghệ in 3D bê tông hoàn toàn có thể cạnh tranh với các công nghệ thi công xây dựng thông thường về tốc độ, tính sẵn có và chi phí.

Máy in 3D bê tông cỡ lớn đầu tiên của Việt Nam, có kích thước tổng thể $4,92 \times 2,53 \times 2,53 \mathrm{~m}$ và kích thước cấu kiện lớn nhất có thể in được lên đến 1,8 x 1,8 x 2,5 m; đã được thiết kế, chế tạo và lắp đặt tại Viện Vật liệu Xây dựng, Bộ Xây dựng. Kết quả nghiên cứu này đã đánh dấu một cột mốc quan trọng trong việc làm chủ một trong các loại hình công nghệ đại diện của ngành xây dựng trong cuộc Cách mạng công nghiệp lần thứ tư tại Việt Nam.

Bài báo này là một phần kết quả nghiên cứu của nhiệm vụ cấp Bộ Xây dựng "Nghiên cứu ứng dụng công nghệ in 3D trong chế tạo vật liệu xây dựng tính năng cao", mã số RD 42-20. Nhóm tác giả trân trọng cảm ơn Bộ Xây dựng đã tạo điều kiện và hỗ trợ chúng tôi hoàn thành nghiên cứu mang tính đột phá, góp phần vào sự phát triển của công nghệ xây dựng trong cuộc Cách mạng công nghiệp lần thứ 4 tại Việt Nam.

\section{Tài liệu tham khảo}

[1]. Izabela Hager, Anna Golonka, Roman Putanowicz. "3D printing of buildings and building components as the future of sustainable construction?", Procedia Engineering 151, p. 292 - 299, 2016. 
[2]. https://www.eecs.psu.edu/news-archive/2019/memari-ali-3d-printedconcrete-system-commercialization.aspx

[3]. Wolfs RJM, Bos FP, Salet TAM, "Early age mechanical behaviour of 3D printed concrete: Numerical modeling and experimental testing". Cem Concr Res 106:103-116, 2018.

[4]. https://cobod.com/gantry-versus-robotic-arm-systems/

[5]. S. Lim, R.A. Buswell 1 , T.T. Le , S.A. Austin , A.G.F. Gibb , T. Thorpe, "Developments in construction-scale additive manufacturing processes", Automation in Construction, 21, 262-268, 2012.

[6]. Behrokh Khoshnevis, Dooil Hwang, Ke-Thia Yao, Zhenghao Yeh, "Megascale fabrication by contour crafting", International Journal of Industrial and Systems Engineering (IJISE), Vol1(No.3), p.301-320, 2006.

[7]. Giovanni Cesaretti, Enrico Dini, Xavier De Kestelier, Valentina Colla, Laurent Pambaguian, "Building components for an outpost on the Lunar soil by means of a novel 3D printing technology". Acta Astronautica, 93, 430-450, 2014.

[8]. De Kestelier, Xavier; Buswell, Richard, "A Digital Design Environment for Large- Scale Rapid Manufacturing”. ACADIA 09: reForm( ) - Building a Better Tomorrow, pp. 201-208, 2009.
[9]. Buswell, R.A.; Leal de Silva, W.R.; Jones, S.Z.; Dirrenberger, J, "3D printing using concrete extrusion: A roadmap for research". Cement and Concrete Research. 112: 37-49, 2018.

[10]. Gosselin, C.; Duballet, R.; Roux, Ph.; Gaudillière, N.; Dirrenberger, J.; Morel, P. "Large-scale 3D printing of ultra-high performance concrete - a new processing route for architects and builders". Materials \& Design. 100: 102109, 2016.

[11]. Le Trung Thanh; Austin, S. A.; Lim, S.; Buswell, R. A.; Gibb, A. G. F.; Thorpe, T. "Mix design and fresh properties for high-performance printing concrete". Materials and Structures. 45 (8): 1221-1232, 2012.

[12]. 3D Systems Corporation, "3D Printing Market - Growth, Trends, COVID-19 Impact, and Forecasts (2021 - 2026)", 2021

[13]. Z.Y. Ahmed, F.P. Bos, M.C.A.J. van Brunschot \& T.A.M. Salet, "On-demand additive manufacturing of functionally graded concrete", Virtual and Physical Prototyping, 2020.

[14]. Bas Wijnen, Gerald C. Anzalone, Amberlee S. Haselhuhn, P. G. Sanders, and J. M. Pearce, "Free and Open-source Control Software for 3-D Motion and Processing" Journal of open research software, no. 2, pp. 1-12, 2016. 